\title{
Whole exome sequencing identifies TRIOBP pathogenic variants as a cause of post- lingual bilateral moderate-to-severe sensorineural hearing loss
}

Agnieszka Pollak ${ }^{1}$, Urszula Lechowicz ${ }^{1}$, Victor Abel Murcia Pieńkowski ${ }^{2,3}$, Piotr Stawiński ${ }^{1}$, Joanna Kosińska², Henryk Skarżyński ${ }^{4}$, Monika Ołdak ${ }^{1 *}$ and Rafał Płoski ${ }^{2^{*}}$

\begin{abstract}
Background: Implementation of whole exome sequencing has provided unique opportunity for a wide screening of causative variants in genetically heterogeneous diseases, including nonsyndromic hearing impairment. TRIOBP in the inner ear is responsible for proper structure and function of stereocilia and is necessary for sound transduction.

Methods: Whole exome sequencing followed by Sanger sequencing was conducted on patients derived from Polish hearing loss family.

Results: Based on whole exome analysis, we identified two TRIOBP pathogenic variants (c.802_805delCAGG, p. GIn268Leufs*610 and c.5014G>T, p.Gly1672*, the first of which was novel) causative of nonsyndromic, peri- to postlingual, moderate-to-severe hearing loss in three siblings from a Polish family. Typically, TRIOBP pathogenic variants lead to prelingual, severe-to-profound hearing loss, thus the onset and degree of hearing impairment in our patients represent a distinct phenotypic manifestation caused by TRIOBP variants. The pathogenic variant $p$. GIn268Leuf**610 disrupts the TRIOBP-4 and TRIOBP-5 isoforms (both expressed exclusively in the inner ear and retina) whereas the second pathogenic variant c.514G>T, p.Gly1672* affects only TRIOBP-5.

Conclusions: The onset and degree of hearing impairment, characteristic for our patients, represent a unique phenotypic manifestation caused by TRIOBP pathogenic variants. Although TRIOBP alterations are not a frequent cause of hearing impairment, this gene should be thoroughly analyzed especially in patients with a postlingual hearing loss. A delayed onset of hearing impairment due to TRIOBP pathogenic variants creates a potential therapeutic window for future targeted therapies.
\end{abstract}

Keywords: Whole exome sequencing, Hearing impairment, TRIOBP

\section{Background}

Two-thirds of early onset hearing impairment (HI) cases are due to genetic causes [1]. After excluding the GJB2 and GJB6 pathogenic variants more than 90 genes may be involved in HI pathogenesis (http://hereditaryhearingloss.org). Massive screening for causative variants within all protein-coding sequences is now available

\footnotetext{
* Correspondence: m.oldak@ifps.org.pl; rploski@wp.pl

'Department of Genetics, Institute of Physiology and Pathology of Hearing, Mochnackiego 10, Warsaw 02-042, Poland

2Department of Medical Genetics, Warsaw Medical University, Pawinskiego

3c, Warsaw 02-106, Poland

Full list of author information is available at the end of the article
}

through whole exome sequencing (WES). Thus, a wide and unbiased search for pathogenic variants has become possible in many genetically heterogeneous diseases including $\mathrm{HI}[2]$.

TRIOBP (Trio- and f-actin-binding protein) (MIM 609761) was cloned in 2001 as TARA [3] and renamed after its mapping to chromosome 22q13.1 [4]. Multiple isoforms of the protein, differing in total length and expression pattern, have been discovered [5, 6]. Both, human and mouse isoforms are classified into long (TRIOBP-3, TRIOBP-5, TRIOBP-6) and short (TRIOBP-1, TRIOBP-2, TRIOBP-4). Interestingly, no part of the 
protein is shared between TRIOBP-1 and TRIOBP-4 [3]. Such a variety of isoforms derived from a single gene may be explained by the presence of six putative alternative promoters [7].

While TRIOBP-1 is widely expressed in different tissues, TRIOBP-4 and TRIOBP-5 was exclusively found in the adult inner ear and retina of both human and mice. In the inner ear TRIOBP-4 and TRIOBP-5 are expressed in stereocilia rootlets. Additionally, TRIOBP-4 is also localized along the whole length of stereocilia. Proper structure of the rootlets is essential for stereocilia rigidity and stiffness, thereby allowing normal process of sound transmission [8]. In contrast, TRIOBP-1 plays a role in regulation of adherens junctions as well as reorganization of the actin cytoskeleton, especially in stress fibers and cortical F-actin [6].

We present here a family with isolated, perilingual to postlingual hearing loss with a recessive inheritance pattern, in which WES followed by direct Sanger sequencing allowed to identify one novel (c.802_805delCAGG, p.Gln268Leufs"610) and one known (c.5014G>T, Gly1672* [9]) TRIOBP pathogenic variant and establish a molecular diagnosis.

\section{Methods}

Pedigree of the analyzed family is presented in Fig. 1a. The level of hearing loss was determined based on pure tone audiometry (PTA) and classified as perilingual if its onset was between 3 to $6 y$ or postlingual with an onset after $6 \mathrm{y}$.

DNA was isolated from peripheral blood by a standard salting out method [10]. In the affected siblings GJB2 pathogenic variants and GJB6 deletions were excluded [11]. Library for WES was prepared with TruSeq Exome Enrichment Kit according to the manufacturer's protocol (Illumina Inc., San Diego, CA, USA), subsequently the sample was run on HiSeq 1500 (Illumina Inc.) with $2 \times$ 100 bp paired-end reads. After obtaining the coverage of minimum 20 times on at least $80 \%$ of the target, bioinformatics analysis was performed as previously described [12]. All retrieved variants were annotated with Annovar and presented in MS Access database for further expert investigations. The DNA sequences were viewed with Integrative Genomic Viewer (IGV) [13, 14].

Potentially pathogenic variants in TRIOBP were confirmed by Sanger sequencing with the use of dedicated primers (sequences available upon request) designed with the Primer 3 software [15] based on the NM_001039141.2 reference sequence.

The prediction of presence of nonsense-mediated mRNA decay (NMD) mechanism was performed with the Mutation Taster algorithm [16].

Exome Aggregation Consortium (ExAC) (http://exac.broadinstitute.org) (accessed 12/2016) and the in-house database of WES data from the Polish population $(n=$ 1052) were used for the assessment of the population prevalence of the detected variants.

\section{Results}

\section{Phenotypic manifestation}

Proband (III.2), as well as the two affected siblings suffered from bilateral, non-syndromic HI. Apart from HI, all of the siblings were otherwise healthy. The age of HI onset was $12 \mathrm{y}$ for the proband and 4,5 y and $3 \mathrm{y}$ for the sibling III.1 and III.3, respectively. The proband was reported for an audiology consultation due to suspected hearing loss based on the parents' observation that she listened to electronic devices on a loud volume. The reason for the audiology tests in the siblings was similar, but it cannot be excluded that the parents were more attentive in observing their younger son. All siblings suffered from moderate-to-severe HI, detailed audiometric data are presented in Fig. 1b. The 2-year follow-up does not show hearing deterioration in sibling III.1 and III.2, for the patient III.3 the additional audiometric data were not available. Notably, the youngest brother (III.3) passed the newborn hearing screening test, the remaining sibs did not have this test. Patient III.3 suffers from frequent otitis media and he has a relevant airbone gap in the right ear $(\sim 40 \mathrm{~dB})$. Now his $\mathrm{HI}$ is classified as mixed comprising of a conductive and sensorineural component.

\section{Variants detection}

WES performed in patient III.2 revealed the presence of two, potentially pathogenic variants in the TRIOBP gene (NM_001039141.2:c.802_805delCAGG, NP_001034230.1: p.Gln268Leufs*610; NM_001039141.2:c.5014G>T, NP_00 1034230.1:p.Gly1672*) both in a heterozygous form (Fig. 1c). The variant c.802_805delCAGG, p.Gln268 Leufs*610 was novel, whereas c.5014G>T, NP_0010 34230.1:p.Gly1672* was already known [9]. Direct Sanger sequencing of the region encompassing the detected variants confirmed the presence of both variants in the proband and her affected siblings. Analysis of parents' DNA showed that they were both heterozygous carriers, thus establishing an in trans configuration of the variants in the affected children (Fig. 1a). The c.802_805delCAGG, p.Gln268Leufs*610 mutation occurs with an allele frequency of 0,000008 and 0,00095 in ExAC and the Polish in-house WES databases, respectively. Allele frequency for c.5014G>T, p.Gly1672*, the second identified TRIOBP variant, was 0,0006 and 0,002 in the same databases (Table 1).

The c.802_805delCAGG, p.Gln268Leufs*610 variant (localized within exon 7) introduces a 4-nucleotide deletion, which results in a frameshift, leading to shortening of the protein after the following 610 amino acids, while 


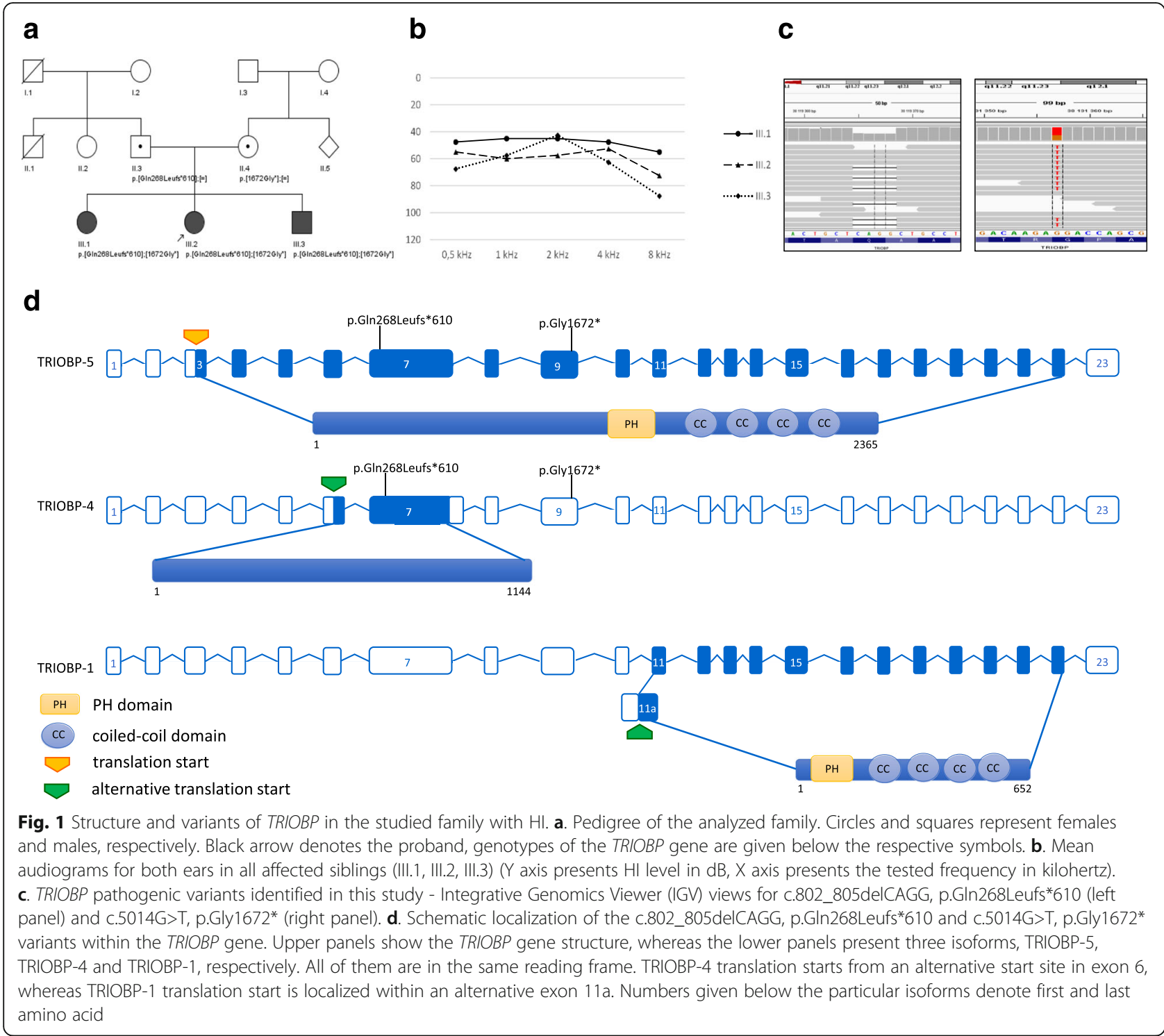

the c.5014G>T, p.Gly1672* variant (localized within exon 9) directly introduces a stop codon. Presence of two deleterious TRIOBP variants in a trans configuration in all affected children strongly suggest that they represent a molecular cause of $\mathrm{HI}$ in these patients.

\section{Discussion}

Here we report on the first TRIOBP family suffering from perilingual or postlingual, moderate-to-severe HI. At the time of writing the onset and degree of HI, characteristic for our patients, represent a unique phenotypic manifestation caused by TRIOBP pathogenic variants. Most of the heretofore detected TRIOBP pathogenic variants are related to prelingual, severe-to-profound hearing loss. There is only one variant (c.154G>A, p.Asp52Asn) assigned to postlingual onset of HI [17] but in this patient the level of HI has not been given. On the other hand, there are few reports on a less severe $\mathrm{HI}$ in patients with TRIOBP variants but with a prelingual onset [18] (Table 1). Thus, our TRIOBP family is the first one with a less severe $\mathrm{HI}$ in respect to both its onset and level.

To date, little is known about the exact function of TRIOBP. It contains two types of relevant domains, i.e. $\mathrm{N}$-terminal Pleckstrin Homology $(\mathrm{PH})$ and $\mathrm{C}$-terminal coiled-coil. It is established that this protein directly binds and stabilizes the F-actin structures [3], presumably via nonconventional actin-binding sites localized in the coiled-coil domains or PH domains $[19,20]$. As its name suggests, Triobp interacts with Trio, a protein derived from a group of Dbl-homology guanine nucleotide exchange factors (DH-GEFs) [3]. DH-GEFs family of 


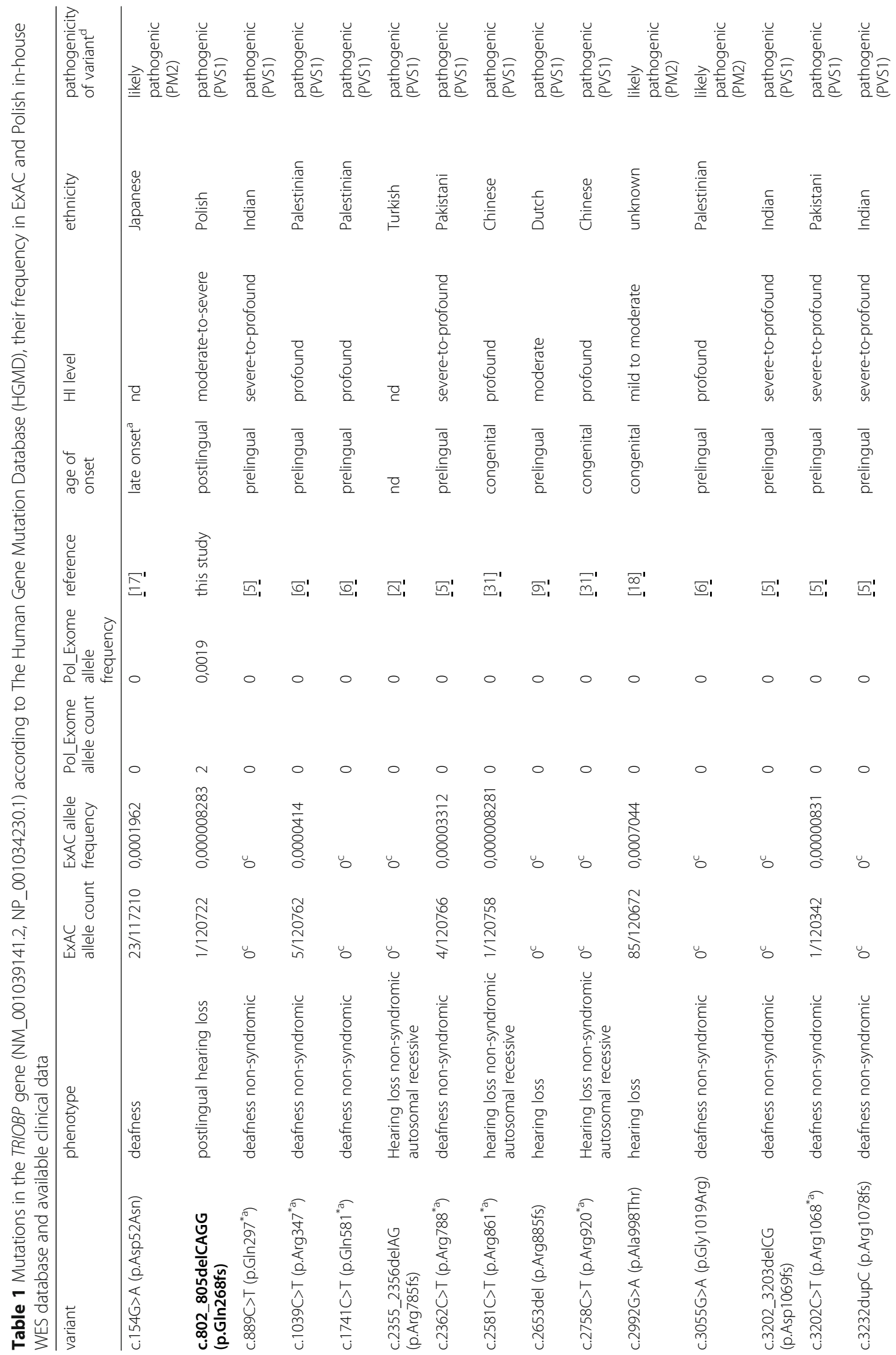


Pollak et al. BMC Medical Genetics (2017) 18:142

Page 5 of 9

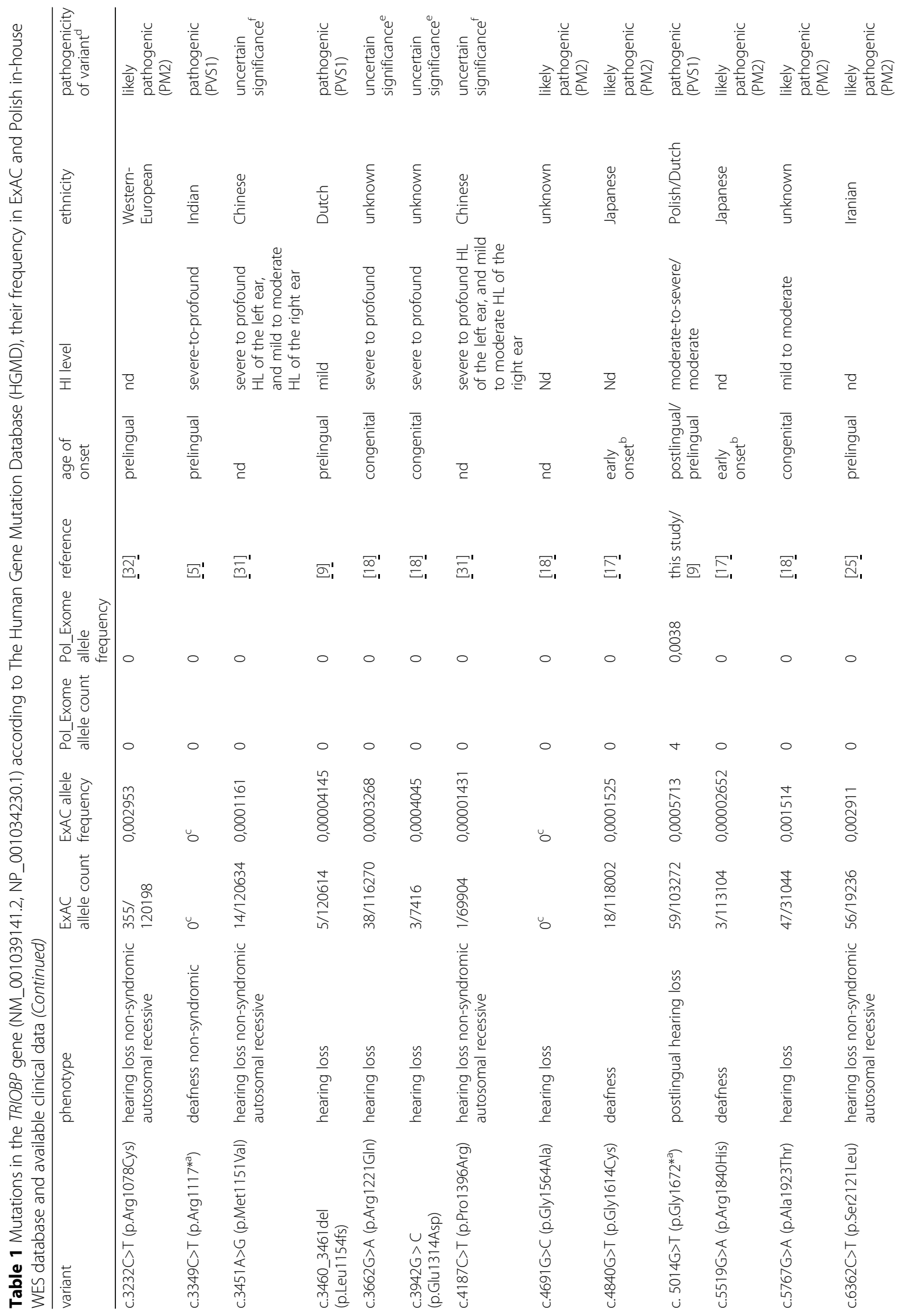




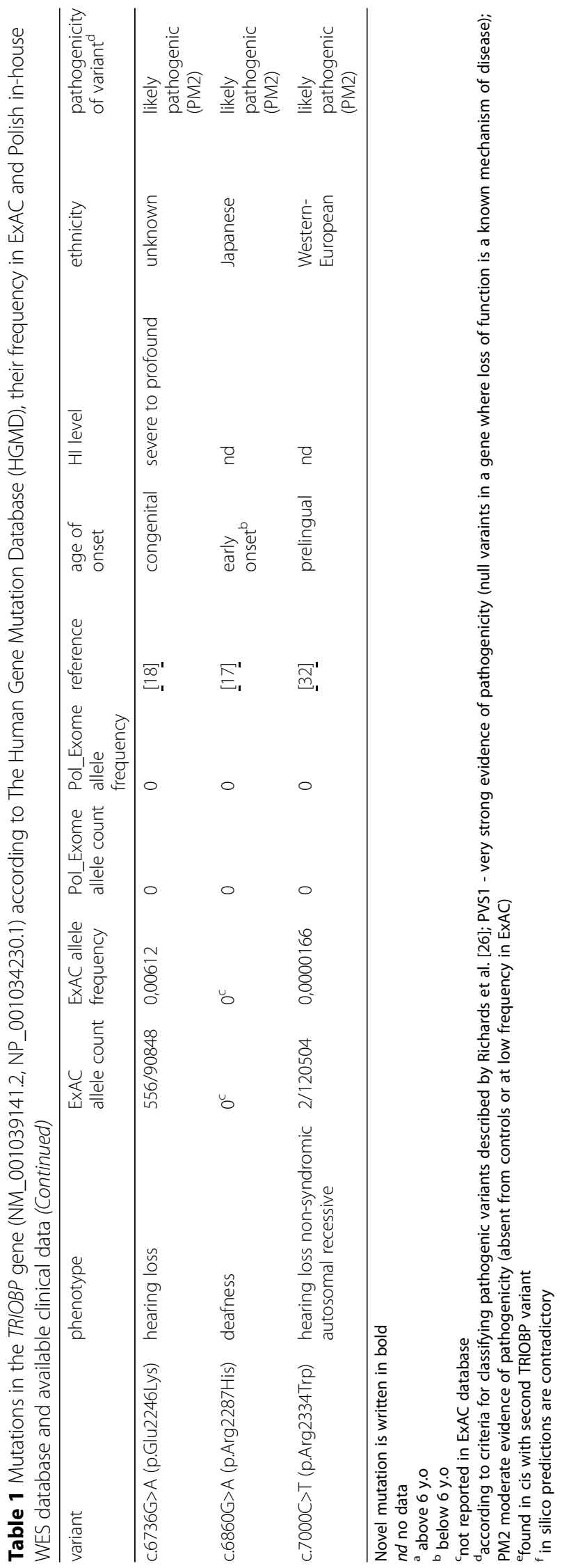


proteins controls reorganization of actin cytoskeleton, cell adhesion, and also serves as transcription factors due to activation of Rho GTPases [21]. Multiple roles of TRIOBP, such as its involvement in organization of actin-cytoskeleton [3], proper centrosomal localization and segregation of chromosomes during cell division [22], cell cycle regulation in conjunction with HECTD3 protein [23] or regulation of cancer cells motility [24] raise the issue why pathogenic variants in this gene do not lead to other pathologies than isolated hearing loss. In the family presented here, both detected variants affects TRIOBP-5, whereas c.802_805deCAGG, p.Gln268 Leufs"610 affects also TRIOBP-4, without impairing the most widely expressed TRIOBP-1 (Fig. 1d), which may explain the selectivity of symptoms. On the other hand, recently some likely pathogenic variants affecting also TRIOBP-1 were described (e.g. homozygous c.6362C > T, p.Ser2121Leu [25]), leading to bilateral, prelingual HI with no other symptoms, thus the comprehensive explanation of this phenotypic phenomena needs further study.

Both variants detected in this study are predicted to result in a premature termination of translation (after amino acid 878 and 1672, respectively). Shortened TRIOPB protein due to c.802_805deCAGG, p.Gln268 Leufs*610 and c.5014G>T, p.Gly1672* variants is devoid of $\mathrm{PH}$ and coiled-coil domains crucial for the actinbinding process. There is also a possibility that the impaired TRIOBP transcripts are directed to degradation via the NMD mechanism, which was suggested by the Mutation Taster algorithm [16]. The conclusive verification of this prediction needs further study; however, due to a restricted expression of TRIOBP-5 and TRIOBP-4 only to the inner ear and the retina we could not test the processing of TRIOBP transcripts in our patients. According to the Criteria for Classifying Pathogenic Variants described by Richards et al. [26], both variants have been classified into a group of very strong evidence of pathogenicity (PVS1) based on their type (nonsense and frameshift), localization within a gene where loss of function is a known mechanism of HI, and low allelic frequency in the ExAC and Polish in-house databases. Additionally, a paper recently published by Wesdorp et al. [9] confirms the pathogenicity of the c.5014G $>$ T, p.Gly1672* variant. Taking into account all the above and the fact that both variants were found in all three affected siblings, we consider c.802_805deCAGG, p.Gln268Leufs"610 and c.5014G>T, p.Gly1672* as causative for HI.

A putative consequence of the described variants is a faulty or absent (via NMD) TRIOBP protein, presumably incapable to form stereocilia rootlets. In response to sound stimulation stereocilia dispossessed of their rootlets, deflect two-four times more intensely than the proper ones and are vulnerable to damage due to their floppiness [27]. These features are consistent with the phenotype observed in the studied family - perilingual or postlingual, moderate-to-severe hearing loss. Furthermore, the localization of the c.5014G $>$ T, p.Gly1672* affecting only the TRIOBP-5 isoform let us assume that the remaining dose of the TRIOBP-4 protein may allow an impaired functioning of the stereocilia, thus leading to a less severe phenotype than those when both isoforms are affected. This is in line with the results of Kitajiri et al. showing that TRIOBP-4 alone is sufficient to form condensed bundles from filamentous-actin parallel to stereocilia rootlets [8]. Similar, milder phenotype (moderate HI) was reported by Wesdorp et al. [9] in a family harboring pathogenic variants: c.2653del, p.Arg885fs*120 and c.5014G $>$ T, p.Gly1672*, although in this case the HI was congenital. It is worth noting that one of the here described siblings passed the newborn screening test (unfortunately the other affected siblings were not tested), which strongly supports that the trigger mechanism damaging hair cells occurs after birth. Considering the role of stereocilia rootlets in adaptation to mechanical stress due to acoustic trauma [27] it is possible that patients harboring truncating variants in the TRIOBP gene are more prone to acoustic trauma. Thus, it seems essential for TRIOBP patients to rigorously avoid noise. On the other hand, a confirmed postnatal origin of HI provides a therapeutic window for targeted treatment in the future.

TRIOBP variants are not a frequent cause of $\mathrm{HI}$. To date, 31 point alterations were described (Table 1) in the TRIOBP gene, with $\mathrm{HI}$ being the only phenotypic manifestation. Most of heretofore described pathogenic or likely pathogenic variants are located within exon 7 , thus this region should be considered as hotspot. Accumulation of repetitive sequences within this area of TRIOBP, may render it more prone to mutation than others [28]. Taking into account the frequency of mutated TRIOBP alleles in the ExAC database, c. $5014 \mathrm{G}>\mathrm{T}$, p.Gly $1672 \%$ is the fourth most common pathogenic variant in this gene, additionally this variant is the most prevalent in the Polish population (Table 1). The data highlights the population distinctiveness and necessity of a preliminary population analysis before introducing a diagnostic test. Thus, in a small diagnostic gene panel for $\mathrm{HI}$ at least the regions encompassing common TRIOBP variants (c.6736G $>$ A, p.Glu2246Lys; c.3232dupC, p.Arg1078Cys; c.2992G >A, p.Ala998Thr and c.5014G>T, p.Gly1672*) should be included. Considering their low frequency in ExAC and our Polish in-house WES databases, point variants in the TRIOBP gene seem to be a rare but important cause 
of non-syndromic hearing loss. Given the high heterogeneity of the HI [29], among patients with unknown cause of this disorder, after exclusion of the GJB2 and GJB6 pathogenic variants, wide screening for causative mutations using next generation sequencing is becoming a standard with at least 18 targeted diagnostic HI panels described to date [30].

\section{Conclusions}

Here we report one novel and one known TRIOBP pathogenic variant (c.802_805delCAGG, p.Gln268fs and c.5014G>T, p.Gly1672*) causative of a unique phenotype. The presented variants disrupt isoform: TRIOBP-4, TRIOBP-5 and TRIOBP-5 respectively, which are expressed in the inner ear and form stereocilia rootlets. Although TRIOBP variants are not a frequent cause of hearing loss, this gene should be thoroughly analyzed also in patients with postlingual HI.

\section{Abbreviations}

DH-GEFs: Dbl-homology guanine nucleotide exchange factors; ExAC: Exome Aggregation Consortium; HGMD: The Human Gene Mutation Database; HI: Hearing impairment; PH: N-terminal Pleckstrin Homology; PTA: Pure tone audiometry; TRIOBP: Trio- and f-actin-binding protein; WES: Whole exome sequencing

\section{Acknowledgments}

Not applicable.

\section{Funding}

This work was supported by NCN grant no. 2011/03/D/NZ5/05592.

\section{Availability of data and materials}

All data apart from the complete results of WES are within the manuscript. The data from next-generation sequencing (WES) in the context of Polish law can be used for personal identification. Due to this we do not have permission to deposit such data in public databases, but data can be made available upon request to the corresponding authors.

\section{Authors' contributions \\ AP carried out the molecular genetic studies and wrote the original draft. UL, VAMP, JK carried out the molecular genetic studies. VAMP, JK, MO, HS performed the literature searching and estimation of the variants pathogenicity. PS participated in the sequence alignment and performed computational analysis. AP, UL, HS, MO participated in phenotyping and clinical data collection. MO, RP wrote, review and edited final version of the manuscript. RP managed the project. All authors read and approved the final manuscript.}

\section{Ethics approval and consent to participate}

The study protocol was approved by Ethical Committee of the Institute of Physiology and Pathology of Hearing (reference number IFPS:/KB/03/2012). Patients or guardians provided informed written consent prior to participation.

\section{Consent for publication}

The patients or their guardians gave written consent for the publication of their medical information.

\section{Competing interests}

The authors declare that they have no competing interests.

\section{Publisher's Note}

Springer Nature remains neutral with regard to jurisdictional claims in published maps and institutional affiliations.

\section{Author details}

'Department of Genetics, Institute of Physiology and Pathology of Hearing, Mochnackiego 10, Warsaw 02-042, Poland. '2Department of Medical Genetics, Warsaw Medical University, Pawinskiego 3c, Warsaw 02-106, Poland.

${ }^{3}$ Postgraduate School of Molecular Medicine, Warsaw Medical University, Warsaw, Poland. ${ }^{4}$ Oto-Rhino-Laryngology Surgery Clinic, Institute of Physiology and Pathology of Hearing, Warsaw/Kajetany, Poland.

Received: 5 February 2017 Accepted: 14 November 2017

Published online: 02 December 2017

\section{References}

1. Morton CC, Nance WE. Newborn hearing screening-a silent revolution. N Engl J Med. 2006;354(20):2151-64.

2. Diaz-Horta O, Duman D, Foster J 2nd, Sirmaci A, Gonzalez M, Mahdieh N, Fotouhi N, Bonyadi M, Cengiz FB, Menendez I, et al. Whole-exome sequencing efficiently detects rare mutations in autosomal recessive nonsyndromic hearing loss. PLoS One. 2012;7(11):e50628.

3. Seipel K, O'Brien SP, lannotti E, Medley QG, Streuli M. Tara, a novel F-actin binding protein, associates with the trio guanine nucleotide exchange factor and regulates actin cytoskeletal organization. J Cell Sci. 2001:114(Pt 2):389-99.

4. Hirosawa M, Nagase T, Murahashi Y, Kikuno R, Ohara O. Identification of novel transcribed sequences on human chromosome 22 by expressed sequence tag mapping. DNA research: an international journal for rapid publication of reports on genes and genomes. 2001:8(1):1-9.

5. Riazuddin S, Khan SN, Ahmed ZM, Ghosh M, Caution K, Nazli S, Kabra M, Zafar AU, Chen K, Naz S, et al. Mutations in TRIOBP, which encodes a putative cytoskeletal-organizing protein, are associated with nonsyndromic recessive deafness. Am J Hum Genet. 2006;78(1):137-43.

6. Shahin H, Walsh T, Sobe T, Abu Sa'ed J, Abu Rayan a, lynch ED, lee MK, Avraham KB, king MC, Kanaan M. mutations in a novel isoform of TRIOBP that encodes a filamentous-actin binding protein are responsible for DFNB28 recessive nonsyndromic hearing loss. Am J Hum Genet. 2006;78(1):144-52

7. Thierry-Mieg D, Thierry-Mieg J: AceView: a comprehensive cDNA-supported gene and transcripts annotation. Genome biology 2006, 7 Suppl 1:S12 11-14.

8. Kitajiri S, Sakamoto T, Belyantseva IA, Goodyear RJ, Stepanyan R, Fujiwara I, Bird JE, Riazuddin S, Riazuddin S, Ahmed ZM, et al. Actin-bundling protein TRIOBP forms resilient rootlets of hair cell stereocilia essential for hearing. Cell. 2010;141(5):786-98.

9. Wesdorp M, van de Kamp JM, Hensen EF, Schraders M, Oostrik J, Yntema HG, Feenstra I, Admiraal RJC, Kunst HPM, Tekin M, et al. Broadening the phenotype of DFNB28: mutations in TRIOBP are associated with moderate stable hereditary hearing impairment. Hear Res. 2017;347:56-62.

10. Miller SA, Dykes DD, Polesky HF. A simple salting out procedure for extracting DNA from human nucleated cells. Nucleic Acids Res. 1988; 16(3):1215.

11. Hoefsloot LH, Roux AF, Bitner-Glindzicz M. Contributors to EDbpm: EMQN best practice guidelines for diagnostic testing of mutations causing nonsyndromic hearing impairment at the DFNB1 locus. European journal of human genetics: EJHG. 2013;21(11):1325-9.

12. Ploski R, Pollak A, Muller S, Franaszczyk M, Michalak E, Kosinska J, Stawinsk P, Spiewak M, Seggewiss H, Bilinska ZT. Does p.Q247X in TRIM63 cause human hypertrophic cardiomyopathy? Circ Res. 2014;114(2):e2-5.

13. Robinson JT, Thorvaldsdottir H, Winckler W, Guttman M, Lander ES, Getz G, Mesirov JP. Integrative genomics viewer. Nat Biotechnol. 2011;29(1):24-6.

14. Wang K, Li M, Hakonarson H. ANNOVAR: functional annotation of genetic variants from high-throughput sequencing data. Nucleic Acids Res. 2010; 38(16):e164.

15. Untergasser A, Cutcutache I, Koressaar T, Ye J, Faircloth BC, Remm M, Rozen SG. Primer3-new capabilities and interfaces. Nucleic Acids Res. 2012;40(15):e115.

16. Schwarz JM, Cooper DN, Schuelke M, Seelow D. MutationTaster2: mutation prediction for the deep-sequencing age. Nat Methods. 2014;11(4):361-2.

17. Miyagawa M, Naito T, Nishio SY, Kamatani N, Usami S. Targeted exon sequencing successfully discovers rare causative genes and clarifies the molecular epidemiology of Japanese deafness patients. PLoS One. 2013;8(8): e71381.

18. Sloan-Heggen $C M$, Bierer $A O$, Shearer $A E$, Kolbe $D L$, Nishimura $C J$, Frees $K L$, Ephraim SS, Shibata SB, Booth KT, Campbell CA, et al. Comprehensive 
genetic testing in the clinical evaluation of 1119 patients with hearing loss Hum Genet. 2016;135(4):441-50.

19. Sobue K, Sellers JR. Caldesmon, a novel regulatory protein in smooth muscle and nonmuscle actomyosin systems. J Biol Chem. 1991;266(19): 12115-8.

20. Yao L, Janmey P, Frigeri LG, Han W, Fujita J, Kawakami Y, Apgar JR, Kawakami T. Pleckstrin homology domains interact with filamentous actin. J Biol Chem. 1999;274(28):19752-61.

21. Rossman KL, Der CJ, Sondek J. GEF means go: turning on RHO GTPases with guanine nucleotide-exchange factors. Nat Rev Mol Cell Biol. 2005;6(2):167-80.

22. Zhu Y, Wang C, Lan J, Yu J, Jin C, Huang H. Phosphorylation of Tara by Plk1 is essential for faithful chromosome segregation in mitosis. Exp Cell Res. 2012;318(18):2344-52.

23. Yu J, Lan J, Zhu Y, Li X, Lai X, Xue Y, Jin C, Huang H. The E3 ubiquitin ligase HECTD3 regulates ubiquitination and degradation of Tara. Biochem Biophys Res Commun. 2008;367(4):805-12.

24. Bao J, Wang S, Gunther LK, Kitajiri S, Li C, Sakamoto T. The actin-bundling protein TRIOBP- 4 and -5 promotes the motility of pancreatic cancer cells. Cancer letters. 2015;356(2 Pt B):367-73.

25. Fardaei M, Sarrafzadeh S, Ghafouri-Fard S, Miryounesi M. Autosomal recessive nonsyndromic hearing loss: a case report with a mutation in TRIOBP gene. International journal of molecular and cellular medicine. 2015; 4(4):245-7.

26. Richards S, Aziz N, Bale S, Bick D, Das S, Gastier-Foster J, Grody WW, Hegde $M, L y o n$ E, Spector E, et al. Standards and guidelines for the interpretation of sequence variants: a joint consensus recommendation of the American College of Medical Genetics and Genomics and the Association for Molecular Pathology. Genet Med. 2015;17(5):405-24.

27. Boutet de Monvel J, Petit C. Wrapping up stereocilia rootlets. Cell. 2010; 141(5):748-50.

28. Viguera E, Canceill D, Ehrlich SD. Replication slippage involves DNA polymerase pausing and dissociation. EMBO J. 2001;20(10):2587-95.

29. Lenz DR, Avraham KB. Hereditary hearing loss: from human mutation to mechanism. Hear Res. 2011;281(1-2):3-10.

30. Ołdak M. Chapter 8 - next generation sequencing in vision and hearing impairment. In: Clinical applications for next-generation sequencing. Boston: Academic Press; 2016. p. 153-70.

31. Gu X, Guo L, Ji H, Sun S, Chai R, Wang L, Li H. Genetic testing for sporadic hearing loss using targeted massively parallel sequencing identifies 10 novel mutations. Clin Genet. 2015;87(6):588-93.

32. Sommen M, Schrauwen I, Vandeweyer G, Boeckx N, Corneveaux JJ, van den Ende J, Boudewyns A, De Leenheer E, Janssens S, Claes K, et al. DNA diagnostics of hereditary hearing loss: a targeted resequencing approach combined with a mutation classification system. Hum Mutat. 2016;37(8):812-9.

\section{Submit your next manuscript to BioMed Central and we will help you at every step:}

- We accept pre-submission inquiries

- Our selector tool helps you to find the most relevant journal

- We provide round the clock customer support

- Convenient online submission

- Thorough peer review

- Inclusion in PubMed and all major indexing services

- Maximum visibility for your research

Submit your manuscript at www biomedcentral.com/submit

) Biomed Central 